\title{
A NEW CLASS OF CONTINUED FRACTION EXPANSIONS FOR THE RATIOS OF HEINE FUNCTIONS $\left({ }^{1}\right)$
}

\author{
BY \\ EVELYN FRANK
}

1. Introduction. A new class of continued fraction expansions of the type

$$
1+\frac{d_{1} z}{f_{1} z+1}+\frac{d_{2} z}{f_{2} z+1}+\frac{d_{3} z}{f_{3} z+1}+\cdots
$$

for the ratios of two "contiguous" $\left(^{2}\right)$ Heine functions $[4 ; 5 ; 6]\left({ }^{3}\right)$, is described here in detail.

The Heine function $\left({ }^{4}\right)$ is the infinite series

$$
\begin{aligned}
\phi(a, b, c, u, z)= & 1+\frac{\left(1-e^{u a}\right)\left(1-e^{u b}\right)}{\left(1-e^{u}\right)\left(1-e^{u c}\right)} z \\
& +\frac{\left(1-e^{u a}\right)\left(1-e^{u(a+1)}\right)\left(1-e^{u b}\right)\left(1-e^{u(b+1)}\right)}{\left(1-e^{u}\right)\left(1-e^{2 u}\right)\left(1-e^{u c}\right)\left(1-e^{u(c+1)}\right)} z^{2}+\cdots,
\end{aligned}
$$

where $u \neq 2 n \pi i /(p+1), c \neq-p \pm 2 n \pi i / u, n, p=0,1,2, \cdots$. Except for these conditions, $a, b, c$, and $u$ are arbitrary. If $a$ or $b$ is equal to $-p \pm 2 n \pi i / u,(1.2)$ reduces to a polynomial. The radius of convergence of (1.2) is 1 if $\left|e^{u}\right|<1$ and is $\left|e^{u(c+1-a-b)}\right|$ if $\left|e^{u}\right|>1$. For simplification in the notation,

$$
e^{u}=q
$$

is used throughout from this point on. With this substitution, the function (1.2) is denoted by $\phi(a, b, c, q, z)$.

The well-known continued fraction of Heine for the ratio of two Heine

Presented to the Society August 23, 1956; received by the editors November 29, 1956.

(1) Research sponsored by the Office of Ordnance Research, U. S. Army, under Contract No. DA-11-022-ORD-2196.

(2) Gauss [3] considered the functions $F(a, b, c, z)$ and called them "contiguous" if the values of one of the first three elements differed by unity. Heine $[6$, p. 102] called them "verwandte Reihe."

(3) Numbers in brackets refer to the bibliography at the end of the paper.

(4) Professor Perron [7] uses $e^{u}$ instead of $q$, which Heine used, for the reason that $q^{a}$, for example, is not single valued. However, by the use of $e^{\text {ua }}$, which is single valued, one does not need to specify the branch of $q^{a}$. However, since this paper was written, Professor Perron's third edition, volume II, of Die Lehre von den Kettenbrïchen, has been published (Teubner, Stuttgart, 1957). In this new edition Professor Perron again uses $q$ instead of $e^{\boldsymbol{x}}$ (cf. p. 125, formula (19)). 
functions $[4 ; 5 ; 6]$, is $\left(^{5}\right)$

$$
\begin{gathered}
\frac{\phi(a, b, c, q, z)}{\phi(a, b+1, c+1, q, z)} \sim 1+\frac{a_{1} z}{1}+\frac{a_{2} z}{1}+\frac{a_{3} z}{1}+\cdots, \\
a_{2 p-1}=-q^{b+p-1} \cdot \frac{\left(1-q^{a+p-1}\right)\left(1-q^{c-b+p-1}\right)}{\left(1-q^{c+2 p-2}\right)\left(1-q^{c+2 p-1}\right)}, \\
a_{2 p}=-q^{a+p-1} \cdot \frac{\left(1-q^{b+p}\right)\left(1-q^{c-a+p}\right)}{\left(1-q^{c+2 p-1}\right)\left(1-q^{c+2 p}\right)}, \quad p=1,2, \cdots .
\end{gathered}
$$

If the real part of $u$ is not zero, or $|q| \neq 1$ by (1.3), (1.4) represents a meromorphic function which is equal to the quotient $\phi(a, b, c, q, z): \phi(a, b+1$, $c+1, q, z)[7$, p. 354]. In the neighborhood of the origin it is equal to the corresponding power series, and furnishes the analytic continuation of the corresponding power series throughout the finite $z$-plane. The equivalence $(\sim)$ sign can therefore be replaced by the equality $(=)$ sign in (1.4). The Heine formula contains the Gauss continued fraction [3] as a special case, where $\lim u=0$ [7, p. 315].

In an earlier paper [2], the author derived and discussed expansions of the type (1.1) for the ratios of two Gauss functions [3]. In this paper, new continued fraction expansions of the form (1.1) are derived for the ratios $\phi(a, b, c, q, z): \phi(a, b+1, c+1, q, z)$ (formula (2.1)), $\phi(a, b, c, q, z): \phi(a+1, b$, $c+1, q, z$ ) (formula $\left(2.1^{\prime}\right)$, and for other similar ratios in which the elements differ by 1 , also for $\phi(a, b, c, q, z): \phi(a, b, c, q, q z)$ (formula (2.9)(iii)). In §3, the regions of convergence for these continued fractions are studied. It is found that each continued fraction converges inside a certain circular region about the origin, dependent on the values of $a, b, c$, and $q$, to the ratio of the two Heine functions which generates the expansion. Exterior to this circular region the continued fraction converges to a ratio of two different Heine functions. In $\$ 4$, certain special continued fraction expansions obtained from (2.1) are considered.

2. New expansions of the type (1.1) for the ratios of two contiguous Heine functions. The ratio of the two Heine functions to which the continued fraction (1.4) converges can be expanded into a continued fraction of the form (1.1), namely,

$$
\begin{gathered}
\frac{\phi(a, b, c, q, z)}{\phi(a, b+1, c+1, q, z)} \sim 1+\frac{d_{1} z}{f_{1} z+1}+\frac{d_{2} z}{f_{2} z+1}+\frac{d_{3} z}{f_{3} z+1}+\cdots K_{1}, \\
d_{p}=-q^{b} \cdot \frac{\left(1-q^{a+p-1}\right)\left(1-q^{c+p-1-b}\right)}{\left(1-q^{c+p-1}\right)\left(1-q^{c+p}\right)}, \quad f_{p}=q^{b} \cdot \frac{1-q^{a+p-1-b}}{1-q^{c+p}}, \\
p=1,2, \cdots .
\end{gathered}
$$

(5) The symbol $\sim$ denotes a formal expansion. If at any time a partial numerator vanishes while the corresponding partial denominator does not vanish, the continued fraction breaks off with the preceding term. In this case the symbol $\sim$ can be replaced by the $=$ sign. 
This expansion is obtained by the successive substitution of the Heine identities $[5$, p. 292]

(i) $\phi(a, b+1, c+1, q, z)-\phi(a, b, c, q, z)$

$$
=q^{b} z \cdot \frac{\left(1-q^{a}\right)\left(1-q^{c-b}\right)}{\left(1-q^{c}\right)\left(1-q^{c+1}\right)} \cdot \phi(a+1, b+1, c+2, q, z) .
$$

(ii) $\phi(a, b+1, c, q, z)-\phi(a+1, b, c, q, z)$

$$
=-q^{a} z \cdot \frac{1-q^{b-a}}{1-q^{c}} \cdot \phi(a+1, b+1, c+1, q, z) .
$$

On interchanging $a$ and $b$, since the Heine function (1.2) is symmetric in $a$ and $b$, one obtains

$$
\begin{gathered}
\frac{\phi(a, b, c, q, z)}{\phi(a+1, b, c+1, q, z)} \sim 1+\frac{d_{1} z}{f_{1} z+1}+\frac{d_{2} z}{f_{2} z+1}+\frac{d_{3} z}{f_{3} z+1}+\cdots, \\
d_{p}=-q^{a} \cdot \frac{\left(1-q^{b+p-1}\right)\left(1-q^{c+p-1-a}\right)}{\left(1-q^{c+p-1}\right)\left(1-q^{c+p}\right)}, \quad f_{p}=q^{a} \cdot \frac{1-q^{b+p-1-a}}{1-q^{c+p}}, \\
p=1,2, \cdots .
\end{gathered}
$$

Similarly, the expansion

$$
\begin{gathered}
\frac{\phi(a, b, c, q, z)}{\phi(a+1, b, c, q, z)} \sim 1+\frac{d_{1} z}{f_{1} z+1}+\frac{d_{2} z}{f_{2} z+1}+\frac{d_{3} z}{f_{3} z+1}+\cdots K_{2}, \\
d_{1}=-q^{a} \cdot \frac{1-q^{b}}{1-q^{c}}, \quad d_{p}=-q^{a} \cdot \frac{\left(1-q^{b+p-1}\right)\left(1-q^{c+p-2-a}\right)}{\left(1-q^{c+p-2}\right)\left(1-q^{c+p-1}\right)}, \\
\quad p=2,3, \cdots \\
f_{p}=q^{a} \cdot \frac{1-q^{b+p-1-a}}{1-q^{c+p-1}}, \quad p=1,2, \cdots .
\end{gathered}
$$

is generated by successive substitution in (2.2)(ii) and in the Heine identities [5, p. 292]

(i) $\phi(a+1, b, c, q, z)-\phi(a, b, c, q, z)$

$$
=q^{a} z \cdot \frac{1-q^{b}}{1-q^{c}} \cdot \phi(a+1, b+1, c+1, q, z) .
$$

(ii) $\phi(a+1, b, c+1, q, z)-\phi(a, b, c, q, z)$

$$
=q^{a} z \cdot \frac{\left(1-q^{b}\right)\left(1-q^{c-a}\right)}{\left(1-q^{c}\right)\left(1-q^{c+1}\right)} \cdot \phi(a+1, b+1, c+2, q, z) .
$$

On interchanging $a$ and $b$, one also obtains the expansion 


$$
\begin{aligned}
& \frac{\phi(a, b, c, q, z)}{\phi(a, b+1, c, q, z)} \sim 1+\frac{d_{1} z}{f_{1} z+1}+\frac{d_{2} z}{f_{2} z+1}+\frac{d_{3} z}{f_{3} z+1}+\cdots, \\
& d_{1}=-q^{b} \cdot \frac{1-q^{a}}{1-q^{c}}, \quad d_{p}=-q^{b} \cdot \frac{\left(1-q^{a+p-1}\right)\left(1-q^{c+p-2-b}\right)}{\left(1-q^{c+p-2}\right)\left(1-q^{c+p-1}\right)},
\end{aligned}
$$

$$
p=2,3, \cdots,
$$

$$
f_{p}=q^{b} \cdot \frac{1-q^{a+p-1-b}}{1-q^{c+p-1}}, \quad \quad p=1,2, \cdots
$$

In the formation of the expansions (2.3) and $\left(2.3^{\prime}\right)$, the following relations are found between (2.1) and (2.3), and between $\left(2.1^{\prime}\right)$ and $\left(2.3^{\prime}\right)$, respectively:

$$
\begin{gathered}
\frac{\phi(a-1, b, c, q, z)}{\phi(a, b, c, q, z)}=1-\frac{q^{a-1} z \cdot \frac{1-q^{b}}{1-q^{c}}}{\frac{\phi(a, b, c, q, z)}{\phi(a, b+1, c+1, q, z)}}, \\
\frac{\phi(a, b-1, c, q, z)}{\phi(a, b, c, q, z)}=1-\frac{q^{b-1} z \cdot \frac{1-q^{a}}{1-q^{c}}}{\frac{\phi(a, b, c, q, z)}{\phi(a+1, b, c+1, q, z)}} .
\end{gathered}
$$

By the Heine identity

$$
\begin{aligned}
\phi(a, b, c, q, z)- & \phi(a, b, c+1, q, z) \\
& =q^{c} z \cdot \frac{\left(1-q^{a}\right)\left(1-q^{b}\right)}{\left(1-q^{c}\right)\left(1-q^{c+1}\right)} \cdot \phi(a+1, b+1, c+2, q, z)
\end{aligned}
$$

and (2.2)(i), (ii), and (2.4)(i), one obtains the expansion

$$
\frac{\phi(a, b, c, q, z)}{\phi(a, b, c+1, q, z)} \sim 1+\frac{d_{1} z}{f_{1} z+1}+\frac{d_{2} z}{f_{2} z+1}+\frac{d_{3} z}{f_{3} z+1}+\cdots,
$$

(2.7) $d_{1}=q^{c} \cdot \frac{\left(1-q^{a}\right)\left(1-q^{b}\right)}{\left(1-q^{c}\right)\left(1-q^{c+1}\right)}, \quad d_{p}=-q^{b} \cdot \frac{\left(1-q^{a+p-1}\right)\left(1-q^{c+p-1-b}\right)}{\left(1-q^{c+p-1}\right)\left(1-q^{c+p}\right)}$,

$$
f_{1}=-q^{a} \cdot \frac{1-q^{b}}{1-q^{c+1}}, \quad f_{p}=q^{b} \cdot \frac{1-q^{a+p-1-b}}{1-q^{c+p}},
$$

or, on the interchange of $a$ and $b$,

$$
p=2,3, \cdots,
$$




$$
\frac{\phi(a, b, c, q, z)}{\phi(a, b, c+1, q, z)} \sim 1+\frac{d_{1} z}{f_{1} z+1}+\frac{d_{2} z}{f_{2} z+1}+\frac{d_{3} z}{f_{3} z+1}+\cdots,
$$

$\left(2.7^{\prime}\right) d_{1}=q^{c} \cdot \frac{\left(1-q^{b}\right)\left(1-q^{a}\right)}{\left(1-q^{c}\right)\left(1-q^{c+1}\right)}, \quad d_{p}=-q^{a} \cdot \frac{\left(1-q^{b+p-1}\right)\left(1-q^{c+p-1-a}\right)}{\left(1-q^{c+p-1}\right)\left(1-q^{c+p}\right)}$,

$$
f_{1}=-q^{b} \cdot \frac{1-q^{a}}{1-q^{c+1}}, \quad f_{p}=q^{a} \cdot \frac{1-q^{b+p-1-a}}{1-q^{c+p}}, \quad p=2,3, \cdots .
$$

The following relations hold between (2.1) and (2.7), and between $\left(2.1^{\prime}\right)$ and $\left(2.7^{\prime}\right)$, respectively:

$$
\begin{aligned}
& \frac{\phi(a-1, b, c-1, q, z)}{\phi(a-1, b, c, q, z)}=1+ \frac{q^{c-1} z \cdot \frac{\left(1-q^{a-1}\right)\left(1-q^{b}\right)}{\left(1-q^{c-1}\right)\left(1-q^{c}\right)}}{-q^{a-1} z \cdot \frac{1-q^{b}}{1-q^{c}}+\frac{\phi(a, b, c, q, z)}{\phi(a, b+1, c+1, q, z)}}, \\
& \frac{\phi(a, b-1, c-1, q, z)}{\phi(a, b-1, c, q, z)}=1+\frac{q^{c-1} z \cdot \frac{\left(1-q^{b-1}\right)\left(1-q^{a}\right)}{\left(1-q^{c-1}\right)\left(1-q^{c}\right)}}{-q^{b-1} z \cdot \frac{1-q^{a}}{1-q^{c}}+\frac{\phi(a, b, c, q, z)}{\phi(a+1, b, c+1, q, z)}} .
\end{aligned}
$$

The continued fractions

(i)

$$
\begin{aligned}
& \frac{\phi(a, b, c, q, z)}{\phi(a+1, b+1, c+1, q, z)} \\
& \sim-q^{a} z \cdot \frac{1-q^{b}}{1-q^{c}}+1+\frac{d_{1} z}{f_{1} z+1}+\frac{d_{2} z}{f_{2} z+1}+\cdots,
\end{aligned}
$$

(ii) $\frac{\phi(a, b, c, q, q z)}{\phi(a+1, b+1, c+1, q, z)}$

$$
\begin{aligned}
& \sim-\frac{1-q^{b}}{1-q^{c}} \cdot z+1+\frac{d_{1} z}{f_{1} z+1}+\frac{d_{2} z}{f_{2} z+1}+\cdots, \\
& \text { (iii) } \frac{\phi(a, b, c, q, z)}{\phi(a, b, c, q, q z)} \\
& \sim 1+\frac{\frac{\left(1-q^{a}\right)\left(1-q^{b}\right)}{1-q^{c}} \cdot z}{-\frac{1-q^{b}}{1-q^{c}} \cdot z+1}+\frac{d_{1} z}{f_{1} z+1}+\frac{d_{2} z}{f_{2} z+1}+\cdots, \\
& d_{p}=-q^{b} \cdot \frac{\left(1-q^{a+p}\right)\left(1-q^{c+p-1-b}\right)}{\left(1-q^{c+p-1}\right)\left(1-q^{c+p}\right)}, \quad f_{p}=q^{b} \cdot \frac{1-q^{a+p-b}}{1-q^{c+p}}, \quad p=1,2, \cdots,
\end{aligned}
$$


are generated by successive substitution of the Heine identities (2.2) (i), (ii), and (2.4)(i). Expansions (2.9)(ii), (iii) follow from (2.9)(i) by the Heine identity $[5$, p. 287]

$$
\begin{aligned}
\phi(a, b, c, q, z)-\phi( & a, b, c, q, q z) \\
& =\frac{\left(1-q^{a}\right)\left(1-q^{b}\right)}{1-q^{c}} z \cdot \phi(a+1, b+1, c+1, q, z) .
\end{aligned}
$$

On the interchange of $a$ and $b$, one obtains the continued fractions

(i) $\frac{\phi(a, b, c, q, z)}{\phi(a+1, b+1, c+1, q, z)}$

$$
\sim-q^{b} z \cdot \frac{1-q^{a}}{1-q^{c}}+1+\frac{d_{1} z}{f_{1} z+1}+\frac{d_{2} z}{f_{2} z+1}+\cdots,
$$

(ii) $\frac{\phi(a, b, c, q, q z)}{\phi(a+1, b+1, c+1, q, z)}$

$$
\sim-\frac{1-q^{a}}{1-q^{c}} z+1+\frac{d_{1} z}{f_{1} z-1}-\frac{d_{2} z}{f_{2} z-1}-\cdots,
$$

$$
\begin{aligned}
& \text { (iii) } \frac{\phi(a, b, c, q, z)}{\phi(a, b, c, q, q z)} \\
& \sim 1+\frac{\frac{\left(1-q^{b}\right)\left(1-q^{a}\right)}{1-q^{c}} \cdot z}{-\frac{1-q^{a}}{1-q^{c}} \cdot z+1}+\frac{d_{1} z}{f_{1} z+1}+\frac{d_{2} z}{f_{2} z+1}+\cdots, \\
& d_{p}=-q^{a} \cdot \frac{\left(1-q^{b+p}\right)\left(1-q^{c+p-1-a}\right)}{\left(1-q^{c+p-1}\right)\left(1-q^{c+p}\right)}, \quad f_{p}=q^{a} \cdot \frac{1-q^{b+p-a}}{1-q^{c+p}}, \quad p=1,2, \cdots .
\end{aligned}
$$

The following identities also hold between (2.9)(i) and (2.1), and between $\left(2.9^{\prime}\right)(\mathrm{i})$ and $\left(2.1^{\prime}\right)$, respectively:

$$
\begin{aligned}
& \text { (2.11) } \frac{\phi(a-1, b, c, q, z)}{\phi(a, b+1, c+1, q, z)}=-q^{a-1} z \cdot \frac{1-q^{b}}{1-q^{c}}+\frac{\phi(a, b, c, q, z)}{\phi(a, b+1, c+1, q, z)}, \\
& \left(2.11^{\prime}\right) \frac{\phi(a, b-1, c, q, z)}{\phi(a+1, b, c+1, q, z)}=-q^{b-1} z \cdot \frac{1-q^{a}}{1-q^{c}}+\frac{\phi(a, b, c, q, z)}{\phi(a+1, b, c+1, q, z)} .
\end{aligned}
$$

Other similar expressions exist between the remaining expansions in this section.

From (2.2)(ii) and (2.1) one obtains the expansion 


$$
\begin{gathered}
\frac{\phi(a+1, b, c, q, z)}{\phi(a, b+1, c, q, z)} \sim 1+\frac{d_{1} z}{f_{1} z+1}+\frac{d_{2} z}{f_{2} z+1}+\frac{d_{3} z}{f_{3} z+1}+\cdots, \\
d_{1}=-q^{b} \cdot \frac{1-q^{a-b}}{1-q^{c}}, \quad d_{p}=-q^{b} \cdot \frac{\left(1-q^{a+p-1}\right)\left(1-q^{c+p-2-b}\right)}{\left(1-q^{c+p-2}\right)\left(1-q^{c+p-1}\right)}, \\
f_{p}=q^{b} \cdot \frac{1-q^{a+p-1-b}}{1-q^{c+p-1}}, \quad p=2,3, \cdots,
\end{gathered}
$$

An analogous expansion for $\phi(a, b+1, c, q, z): \phi(a+1, b, c, q, z)$ can be obtained by the interchange of $a$ and $b$ in the values of $d_{p}$ and $f_{p}$ in (2.12).

Finally, by use of the algorithm of Euler [1, p. 370] for obtaining a continued fraction of the form (1.1) from the corresponding power series, one obtains the expansion

$$
\begin{aligned}
& \frac{1}{\phi(a, b, c, q, z)} \sim 1+\frac{d_{1} z}{f_{1} z+1}+\frac{d_{2} z}{f_{2} z+1}+\frac{d_{3} z}{f_{3} z+1}+\cdots, \\
& d_{p}=-\frac{\left(1-q^{a+p-1}\right)\left(1-q^{b+p-1}\right)}{\left(1-q^{p}\right)\left(1-q^{c+p-1}\right)}=-f_{p}, \quad p=1,2, \cdots,
\end{aligned}
$$

(cf. Heine [5, p. 293]).

The question of the convergence of the expansions in this section is now considered.

3. The convergence of the continued fractions in $\$ 2\left(^{6}\right)$. Expansion (2.1) is first considered. It is a limit-periodic continued fraction of the form (1.1). There are a number of cases to be considered. (i) Let $R(u)<0$, or $\left|e^{u}\right|=|q|$ $<1$. Then $\lim _{p \rightarrow \infty} d_{p} z=-q^{b} z, \lim _{p \rightarrow \infty}\left(f_{p} z+1\right)=q^{b} z+1$, and the roots of the auxiliary equation $x^{2}-\lim _{p \rightarrow \infty}\left(f_{p} z+1\right) x-\lim _{p \rightarrow \infty} d_{p} z=0$ are $q^{b} z$ and 1 . They are of unequal modulus if $|z| \neq\left|q^{-b}\right|$. Then, by Theorems 41 and 42 of Perron $\left[7\right.$, p. 286], (2.1) converges (at least in the "wider sense") if $|z| \neq\left|q^{-b}\right|$. Furthermore, there exist positive numbers $M$ and $n$ such that, for $p \geqq n$, the continued fraction

$$
b_{p} z+1+\frac{a_{p+1} z}{b_{p+1} z+1}+\frac{a_{p+2} z}{b_{p+2} z+1}+\cdots
$$

converges uniformly for $|z|<(1-\epsilon)\left|q^{-b}\right|$ and for $M>z>(1+\epsilon)\left|q^{-b}\right|$, where

${ }^{(6)}$ In any of the expansions in $\$ 2$, for certain values of $a, b$, or $c$, a partial numerator of a continued fraction may be zero. In this case, the continued fraction is finite, and its value can be computed therefrom. Furthermore, it is understood throughout that in each case those values of $a, b$, or $c$ are excluded which make indeterminate the function to which the continued fraction in question converges. 
$\epsilon$ is an arbitrarily small positive number. Then, by a proof entirely analogous to that of Perron [7, p. 342] for Stieltjes type continued fractions, since (3.1) converges uniformly for $|z|<(1-\epsilon)\left|q^{-b}\right|, R(u)<0$, (2.1) is equal to the corresponding power series within the circle $|z|=\left|q^{-b}\right|$. The continued fraction (2.1) thus converges to the function $\phi(a, b, c, q, z) ; \phi(a, b+1, c+1, q, z)$ within $|z|=\left|q^{-b}\right|$ provided $R(u)<0$, or $|q|<1$ by (1.3).

(ii) Let $R(u)>0$, or $\left|e^{u}\right|=|q|>1$. Then $\lim _{p \rightarrow \infty} d_{p} z=-q^{a-c-1} z$, $\lim _{p \rightarrow \infty}\left(f_{p} z+1\right)=q^{a-c-1} z+1$, and the roots of the auxiliary equation are $q^{a-c-1}$ and 1. Therefore, by the same type of proof as in (i), the continued fraction (3.1) converges uniformly for $|z|<(1-\epsilon)\left|q^{c+1-a}\right|$ and for $M_{1}>|z|$ $>(1+\epsilon)\left|q^{c+1-a}\right|$ provided $R(u)>0$, and the continued fraction (2.1) is equal to the corresponding power series within $|z|=\left|q^{c+1-a}\right|$. The continued fraction (2.1) therefore converges to the function

$$
\phi(a, b, c, q, z): \phi(a, b+1, c+1, q, z)
$$

within $|z|=\left|q^{c+1-a}\right|$ if $R(u)>0$, or $|q|>1$ by (1.3).

For the investigation of the functions to which (2.1) converges for $|z|>\left|q^{-b}\right|, R(u)<0$, and $|z|>\left|q^{c+1-a}\right|, R(u)>0$, let (2.1) by an equivalence transformation be written in the form $\left({ }^{7}\right)$

$$
\begin{aligned}
& \frac{1-q^{c}}{q^{b} z\left(1-q^{a-b-1}\right)}\left[K_{1}-1\right]+1 \\
& \quad=\frac{\phi\left(a, a-c-1, a-b-1, q, q^{c+1-a-b} / z\right)}{\phi\left(a, a-c, a-b, q, q^{c+1-a-b} / z\right)}=1+\frac{d_{1}^{\prime} / z}{f_{1}^{\prime} / z+1}+\frac{d_{2}^{\prime} / z}{f_{2}^{\prime} / z+1}+\cdots, \\
& d_{p}^{\prime}=-\frac{\left(1-q^{a+p-1}\right)\left(1-q^{c-b+p-1}\right)}{q^{b}\left(1-q^{a-b+p-2}\right)\left(1-q^{a-b+p-1}\right)}, \quad f_{p}=\frac{1-q^{c+p}}{q^{b}\left(1-q^{a-b+p-1}\right)}, p=1,2, \cdots,
\end{aligned}
$$

where the equality sign has just been proved in (i) and (ii) above to hold for certain values of $|z|$ dependent on $R(u)$. Consequently, the value of $K_{1}$ in (2.1) is

$$
\begin{aligned}
& \frac{q^{b} z\left(1-q^{a-b-1}\right)}{1-q^{c}} \cdot\left[\frac{\phi\left(a, a-c-1, a-b-1, q, q^{c+1-a-b} / z\right)}{\phi\left(a, a-c, a-b, q, q^{c+1-a-b} / z\right)}-1\right]+1 \\
& \quad=-\frac{\left(1-q^{a}\right)\left(1-q^{c-b}\right)}{\left(1-q^{c}\right)\left(1-q^{a-b}\right)} \cdot \frac{\phi\left(a+1, a-c, a-b+1, q, q^{c+1-a-b} / z\right)}{\phi\left(a, a-c, a-b, q, q^{c+1-a-b} / z\right)}+1, \quad \text { by }(2.2)(\mathrm{i}), \\
& \quad=\frac{\left(1-q^{b}\right)\left\{\left(1-q^{a-b}\right) \cdot \phi\left(a, a-c, a-b, q, q^{c+1-a-b} / z\right)-\left(1-q^{c-b}\right) \cdot \phi\left(a, a-c, a-b+1, q, q^{c+1-a-b} / z\right)\right\}}{\left(1-q^{c}\right)\left(1-q^{a-b}\right) \phi\left(a, a-c, a-b, q, q^{c+1-a-b} / z\right)}
\end{aligned}
$$

by the Heine identity (35), [5, p. 291],

(7) The method of proof for convergence exterior to the circles in (i) and (ii) was suggested to the author by Professor Perron. It can also be used to obtain the convergence of the continued fraction expansions for the ratios of two Gauss functions considered by the author in [2]. 


$$
\begin{aligned}
&\left(1-q^{c}\right) \phi(a, b, c, q, z)-\left(1-q^{c-a}\right) \phi(a, b, c+1, q, z) \\
&-q^{c-a}\left(1-q^{a}\right) \phi(a+1, b, c+1, q, z)=0 .
\end{aligned}
$$

Finally, by use of the Heine identity (29), [5, p. 290],

$$
\begin{array}{r}
\left(1-q^{c-b}\right) \phi(a, b, c+1, q, z)+q^{c-b}\left(1-q^{b}\right) \phi(a, b+1, c+1, q, z) \\
-\left(1-q^{c}\right) \phi(a, b, c, q, z)=0,
\end{array}
$$

the value of $K_{1}$ in $(2.1)$ becomes

$$
\frac{\left(1-q^{-b}\right)\left(1-q^{a-c}\right) \phi\left(a, a-c+1, a-b+1, q, q^{c+1-a-b} / z\right)}{\left(1-q^{-c}\right)\left(1-q^{a-b}\right) \phi\left(a, a-c, a-b, q, q^{c+1-a-b} / z\right)}
$$

for $\left|q^{c+1-a-b} / z\right|<\left|q^{c+1-a}\right|$ or $|z|>\left|q^{-b}\right|$ if $R(u)<0$, and for $\left|q^{c+1-a-b} / z\right|$ $<\left|q^{-b}\right|$ or $|z|>\left|q^{c+1-a}\right|$ if $R(u)>0$. On interchanging $a$ and $b$, one likewise obtains the values of $\left(2.1^{\prime}\right)$.

The following theorem has thus been proved.

THEOREM 3.1. The continued fraction (2.1) converges to the value

$$
\phi(a, b, c, q, z): \phi(a, b+1, c+1, q, z)
$$

within the circle $|z|=\left|q^{-b}\right|$ if $R(u)<0$ and within the circle $|z|=\left|q^{c+1-a}\right|$ if $R(u)>0$. It converges to the value

$$
\frac{\left(1-q^{-b}\right)\left(1-q^{a-c}\right) \phi\left(a, a-c+1, a-b+1, q, q^{c+1-a-b} / z\right)}{\left(1-q^{-c}\right)\left(1-q^{a-b}\right) \phi\left(a, a-c, a-b, q, q^{c+1-a-b} / z\right)}
$$

for $|z|>\left|q^{-b}\right|$ if $R(u)<0$, and for $|z|>\left|q^{c+1-a}\right|$ if $R(u)>0$. The continued fraction $\left(2.1^{\prime}\right)$ converges to the value

$$
\phi(a, b, c, q, z): \phi(a+1, b, c+1, q, z)
$$

within the circle $|z|=\left|q^{-a}\right|$ if $R(u)<0$ and within the circle $|z|=\left|q^{c+1-b}\right|$ if $R(u)>0$. It converges to the value

$$
\frac{\left(1-q^{-a}\right)\left(1-q^{b-c}\right) \phi\left(b, b-c+1, b-a+1, q, q^{c+1-a-b} / z\right)}{\left(1-q^{-c}\right)\left(1-q^{b-a}\right) \phi\left(b, b-c, b-a, q, q^{c+1-a-b} / z\right)}
$$

for $|z|>\left|q^{-a}\right|$ if $R(u)<0$, and for $|z|>\left|q^{c+1-b}\right|$ if $R(u)>0$.

In order to investigate the convergence of $(2.3)$, the roots of the auxiliary equation one finds are $v^{a} z$ and 1 if $R(u)<0$. Consequently, (2.3) converges to the function $\phi(a, b, c, q, z): \phi(a+1, b, c, q, z)$ within $|z|=\left|q^{-a}\right|$ if $R(u)<0$, by the same type of proof as in the case of (2.1). If $R(u)>0$, the roots of the auxiliary equation are $q^{b-c} z$ and 1 , so that (2.3) converges to the function $\phi(a, b, c, q, z): \phi(a+1, b, c, q, z)$ within $|z|=\left|q^{c-b}\right|$ if $R(u)>0$.

For the investigation of the convergence of (2.3) exterior to these circles, one transforms (2.3) into 


$$
\begin{array}{r}
\frac{1}{q^{a} z} \cdot\left[\frac{\phi(a, b, c, q, z)}{\phi(a+1, b, c-q, z)}-1\right]+1=\frac{\phi\left(b-c, b, b-a, q, q^{c-a-b} / z\right)}{\phi\left(b-c+1, b, b-a, q, q^{c-a-b} / z\right)} \\
=1+\frac{d_{1}^{\prime} / z}{f_{1}^{\prime} / z+1}+\frac{d_{2}^{\prime} / z}{f_{2}^{\prime} / z+1}+\frac{d_{3}^{\prime} / z}{f_{3}^{\prime} / z+1}+\cdots,
\end{array}
$$

$$
\begin{gathered}
d_{1}^{\prime}=\frac{1-q^{b}}{q^{a}\left(1-q^{b-a}\right)}, d_{p}^{\prime}=-\frac{\left(1-q^{b+p-1}\right)\left(1-q^{c-a+p-2}\right)}{q^{a}\left(1-q^{b-a+p-2}\right)\left(1-q^{b-a+p-1}\right)}, p=2,3, \cdots, \\
f_{p}^{\prime}=\frac{1-q^{c+p-1}}{q^{a}\left(1-q^{b-a+p-1}\right)}, \quad p=1,2, \cdots,
\end{gathered}
$$

where the equality sign holds for the cases stated in the preceding paragraph. From (3.6), by (2.4)(i),

$$
K_{2}=1-\frac{1-q^{b}}{1-q^{b-a}} \cdot \frac{\phi\left(b-c+1, b+1, b-a+1, q, q^{c-a-b} / z\right)}{\phi\left(b-c+1, b, b-a, q, q^{c-a-b} / z\right)}
$$

for $|z|>\left|q^{-a}\right|, R(u)<0$, and for $|z|>\left|q^{c-b}\right|, R(u)>0$. On the interchange of $a$ and $b$, the values of $\left(2.3^{\prime}\right)$ can be determined. The following theorem has thus been proved.

THEOREM 3.2. The continued fraction (2.3) converges to the value

$$
\phi(a, b, c, q, z): \phi(a+1, b, c, q, z)
$$

within the circle $|z|=\left|q^{-a}\right|$ if $R(u)<0$, and within the circle $|z|=\left|q^{c-b}\right|$ if $R(u)>0$. It converges to the value (3.7) for $|z|>\left|q^{-a}\right|$ if $R(u)<0$, and for $|z|>\left|q^{c-b}\right|$ if $R(u)>0$. The continued fraction $\left(2.3^{\prime}\right)$ converges to the value

$$
\phi(a, b, c, q, z): \phi(a, b+1, c, q, z)
$$

within the circle $|z|=\left|q^{-b}\right|$ if $R(u)<0$ and within the circle $|z|=\left|q^{c-a}\right|$ if $R(u)>0$. It converges to the value

$$
1-\frac{\left(1-q^{a}\right) \cdot \phi\left(a-c+1, a+1, a-b+1, q, q^{c-a-b} / z\right)}{\left(1-q^{a-b}\right) \cdot \phi\left(a-c+1, a, a-b, q, q^{c-a-b} / z\right)}
$$

for $|z|>\left|q^{-b}\right|$ if $R(u)<0$, and for $|z|>\left|q^{c-a}\right|$ if $R(u)>0$.

Following the same procedure, by use of (2.2)(i), (2.8), (3.5), and the Heine identity (19), [5, p. 290],

$$
\begin{aligned}
&\left(1-q^{b-a}\right) \phi(a, b, c, q, z)+q^{b-a}\left(1-q^{a}\right) \phi(a+1, b, c, q, z) \\
&-\left(1-q^{b}\right) \phi(a, b+1, c, q, z)=0,
\end{aligned}
$$

one obtains the following theorem on the convergence of (2.7). 
THEOREM 3.3. The continued fraction (2.7) converges to the value $\phi(a, b, c, q, z): \phi(a, b, c+1, q, z)$ within the circle $|z|=\left|q^{-b}\right|$ if $R(u)<0$, and within the circle $|z|=\left|q^{c+1-a}\right|$ if $R(u)>0$. It converges to the value

$\frac{\left(1, q^{a-c}\right) \cdot \phi\left(a, a-c+1, a-b+1, q, q^{c+1-a-b} / z\right)}{\left(1-q^{-c}\right)} \frac{\left.\phi\left(a, a-c, a-b+1, q, q^{c+1-a-b} / z\right)-\left(1-q^{a}\right) \phi\left(a+1, a-c, a-b+1, q, q^{c+1-a-b}\right) / z\right)}{-}$ or

$$
1 /\left[1-\frac{\left(1-q^{a}\right) \cdot \phi\left(a+1, a-c, a-b+1, q, q^{c+1-a-b} / z\right)}{\left(1-q^{a-c}\right) \cdot \phi\left(a, a-c+1, a-b+1, q, q^{c+1-a-b} / z\right)}\right]
$$

for $|z|>\left|q^{-b}\right|$ if $R(u)<0$, and for $|z|>\left|q^{c+1-a}\right|$ if $R(u)>0$. The functions to which the expansion $\left(2.7^{\prime}\right)$ converges as well as the regions of convergence can be obtained from the above with the interchange of $a$ and $b$.

By the relation (2.11), with the help of (2.4)(i) and (3.5), one obtains the following theorem on the convergence of the expansions (2.9).

THEOREM 3.4. The continued fractions (2.9)(i), (ii), (iii), converge to the values

$$
\begin{aligned}
\phi(a, b, c, q, z) & : \phi(a+1, b+1, c+1, q, z), \\
\phi(a, b, c, q, q z) & : \phi(a+1, b+1, c+1, q, z), \\
\phi(a, b, c, q, z) & : \phi(a, b, c, q, q z),
\end{aligned}
$$

respectively, within the circle $|z|=\left|q^{-b}\right|$ if $R(u)<0$, and within the circle $|z|=\left|q^{c-a}\right|$ if $R(u)>0$. Expansion (2.9)(i) converges to the value

$$
\frac{\left(1-q^{b}\right)}{\left(1-q^{c}\right)} \cdot z \cdot\left[-q^{a}+1-\frac{\phi\left(a, a-c+1, a-b+1, q, q^{c-a-b} / z\right)}{\phi\left(a+1, a-c+1, a-b+1, q, q^{c-a-b} / z\right)}\right],
$$

(2.9) (ii) to the value

$$
-\frac{\left(1-q^{b}\right)}{\left(1-q^{c}\right)} \cdot z \cdot \frac{\phi\left(a, a-c+1, a-b+1, q, q^{c-a-b} / z\right)}{\phi\left(a+1, a-c+1, a-b+1, q, q^{c-a-b} / z\right)},
$$

and (2.9) (iii) to the value

$$
\frac{\phi\left(a, a-c+1, a-b+1, q, q^{c-a-b} / z\right)-\left(1-q^{a}\right) \phi\left(a+1, a-c+1, a-b+1, q, q^{c-a-b} / z\right)}{\phi\left(a, a-c+1, a-b+1, q, q^{c-a-b} / z\right)}
$$

for $|z|>\left|q^{-b}\right|$ if $R(u)<0$, and for $|z|>\left|q^{c-a}\right|$ if $R(u)>0$. The functions to which the expansions $\left(2.9^{\prime}\right)$ converge as well as the regions of convergence can be obtained from the above with the interchange of $a$ and $b$.

By the use of (2.2) (ii), (2.4)(ii), and (3.5), one obtains the following theorem on the convergence of the expansion (2.12). 
THEOREM 3.5. The continued fraction (2.12) converges to the value

$$
\phi(a+1, b, c, q, z): \phi(a, b+1, c, q, z)
$$

within the circle $|z|=\left|q^{-b}\right|$ if $R(u)<0$ and within the circle $|z|=\left|q^{c-a}\right|$ if $R(u)>0$. It converges to the value

$$
1-\frac{\phi\left(a+1, a-c+1, a-b+1, q, q^{c-a-b} / z\right)}{\phi\left(a, a-c+1, a-b, q, q^{c-a-b} / z\right)}
$$

for $|z|>\left|q^{-b}\right|$ if $R(u)<0$, and for $|z|>\left|q^{c-a}\right|$ if $R(u)>0$.

An analogous theorem holds for the expansion of $\phi(a, b+1, c, q, z)$ : $\phi(a+1, b, c, q, z)$.

4. Continued fraction expansions for the ratios of specialized Heine functions. Let $b=0$, and $c$ be written for $c+1$. Then (2.1) becomes

$$
\begin{aligned}
& \frac{1}{\phi(a, 1, c, q, z)}=1+\frac{d_{1} z}{f_{1} z+1}+\frac{d_{2} z}{f_{2} z+1}+\frac{d_{3} z}{f_{3} z+1}+\cdots, \\
& d_{p}=-f_{p}=-\frac{1-q^{a+p-1}}{1-q^{c+p-1}}, \quad p=1,2, \cdots, \\
& |z|<1 \text { if } R(u)<0, \quad|z|<\left|q^{c-a}\right| \text { if } R(u)>0,
\end{aligned}
$$

(cf. (5.2) of [2] for a similar expansion of the analogous Gauss function). It is remarked that (4.1) can also be obtained from (2.12) or (2.13).

In analogy to the expansion of the Gauss function $F(a, 1,1, z)$ for $(1-z)^{a}$ (expansion (i), [2, p. 470]), one obtains for the reciprocal of

$$
\phi(a, 1,1, q, z)=1+\frac{1-q^{a}}{1-q} z+\frac{\left(1-q^{a}\right)\left(1-q^{a+1}\right)}{(1-q)\left(1-q^{2}\right)} z^{2}+\cdots,
$$

[6, p. 99], the expansion

$$
\begin{aligned}
& \frac{1}{\phi(a, 1,1, q, z)}=1+\frac{d_{1} z}{f_{1} z+1}+\frac{d_{2} z}{f_{2} z+1}+\frac{d_{3} z}{f_{3} z+1}+\cdots, \\
& d_{p}=-f_{p}=-\frac{1-q^{a+p-1}}{1-q^{p}}, \quad p=1,2, \cdots, \\
& |z|<1 \text { if } R(u)<0, \quad|z|<\left|q^{1-a}\right| \text { if } R(u)>0 .
\end{aligned}
$$

For the reciprocal of

$$
\frac{\phi(1,1,2, q, z)}{1-q}=\frac{1}{1-q}+\frac{z}{1-q^{2}}+\frac{z^{2}}{1-q^{3}}+\cdots,
$$

[6, p. 99], one obtains the expansion 


$$
\begin{gathered}
\frac{1-q}{\phi(1,1,2, q, z)}=\frac{1-q}{1}+\frac{(1-q) \cdot d_{1} z}{f_{1} z+1}+\frac{d_{2} z}{f_{2} z+1}+\frac{d_{3} z}{f_{3} z+1}+\cdots, \\
d_{p}=-f_{p}=-\frac{1-q^{p}}{1-q^{p+1}}, \quad p=1,2, \cdots \\
|z|<1 \text { if } R(u)<0, \quad|z|>|q| \text { if } R(u)>0 .
\end{gathered}
$$

This is analogous to formula (ii), [2, p. 471] for the Gauss function.

For the reciprocal of

$$
\frac{\phi\left(1,1 / 2,3 / 2, q^{2}, z^{2}\right)}{1-q}=\frac{1}{1-q}+\frac{z^{2}}{1-q^{3}}+\frac{z^{4}}{1-q^{5}}+\cdots,
$$

[6, p. 99], from $\left(2.1^{\prime}\right)$ one obtains the expansion

$$
\frac{1-q}{\phi\left(1,1 / 2,3 / 2, q^{2}, z^{2}\right)}=\frac{1-q}{1}+\frac{(1-q) \cdot d_{1} z^{2}}{f_{1} z^{2}+1}+\frac{d_{2} z^{2}}{f_{2} z^{2}+1}+\frac{d_{3} z^{2}}{f_{3} z^{2}+1}+\cdots,
$$

$$
\begin{aligned}
& d_{p}=-f_{p}=-\frac{1-q^{2 p-1}}{1-q^{2 p+1}} \\
& |z|<1 \text { if } q<1, \quad\left|z^{2}\right|<\left|q^{2}\right| \text { if } q>1 .
\end{aligned}
$$

This is analogous to formula (iii), [2, p. 471] for the Gauss function.

\section{Bibliography}

1. L. Euler, De fractionibus continuis, Introductio in analysin infinitorum, vol. 1, Chapter 18, 1748 (Opera Omnia, Series Prima, vol. 8, pp. 362-390).

2. E. Frank, $A$ new class of continued fraction expansions for the ratios of hypergeometric functions, Trans. Amer. Math. Soc. vol. 81 (1956) pp. 453-476.

3. C. F. Gauss, Disquisitiones generales circa seriem infinitam $1+\alpha \beta x / 1 \cdot \gamma+\alpha(\alpha+1)$ $\cdot \beta(\beta+1) x x / 1 \cdot 2 \cdot \gamma(\gamma+1)+\alpha(\alpha+1)(\alpha+2) \beta(\beta+1)(\beta+2) x^{3} / 1 \cdot 2 \cdot 3 \cdot \gamma(\gamma+1)(\gamma+2)+$ etc., Werke, vol. 3 (1876) pp. 125-162.

4. E. Heine, Über die Reihe

$$
1+\left(\frac{\left(q^{\alpha}-1\right)\left(q^{\beta}-1\right)}{(q-1)\left(q^{\gamma}-1\right)}\right) x+\left(\frac{\left(q^{\alpha}-1\right)\left(q^{\alpha+1}-1\right)\left(q^{\beta}-1\right)\left(q^{\beta+1}-1\right)}{(q-1)\left(q^{2}-1\right)\left(q^{\gamma}-1\right)\left(q^{\gamma+1}-1\right)}\right) x^{2}+\cdots,
$$

Jrn. für Math. vol. 32 (1846) pp. 210-212.

5. - Untersuchungen ïber die Reihe

$$
1+\left(\frac{\left(1-q^{\alpha}\right)\left(1-q^{\beta}\right)}{(1-q)\left(1-q^{\gamma}\right)}\right) x+\left(\frac{\left(1-q^{\alpha}\right)\left(1-q^{\alpha+1}\right)\left(1-q^{\beta}\right)\left(1-q^{\beta+1}\right)}{(1-q)\left(1-q^{2}\right)\left(1-q^{\gamma}\right)\left(1-q^{\gamma+1}\right)}\right) x^{2}+\cdots,
$$

Jrn. für Math. vol. 34 (1847) pp. 285-328.

6. - Handbuch der Kugelfuncktionen, vol. 1, Berlin, Reimer, 1878.

7. O. Perron, Die Lehre von den Kettenbrilchen, Leipzig, Teubner, 1929.

UNIVERSITY OF ILLINOIS,

Chicago, Ill. 